TRANSACTIONS OF THE

AMERICAN MATHEMATICAL SOCIETY

Volume 281. Number I, January 1984

\title{
SETS OF ESSENTIALLY UNITARY OPERATORS
}

BY

RIDGLEY LANGE

\begin{abstract}
Let $U_{e}$ be the set of essentially unitary operators on a separable Hilbert space $H$; for $1 \leqslant p \leqslant \infty$, let $U_{p}$ be the set of operators $T$ such that $I-T^{*} T$ lies in the Schatten $p$-ideal and the spectrum of $T$ does not fill the unit disc: and let $U_{c}^{\prime \prime}$ be the set of operators in $U_{e}$ of Fredholm index $n$. The author proves that each $U_{c}^{n}$ is closed and path connected, that $U_{p}$ is dense in $U_{c}^{(0)}$ and $U_{p}$ is path connected for each $p$, and that all these sets are invariant under Cayley transform. It is proved that the spectrum is continuous on $U_{x}$ but not on $U_{e}$, while the spectral radius is continuous on $U_{c}$. Sufficient conditions that an operator in $U_{e}$ have a nontrivial hyperinvariant subspace are given, and it is proved that the general hyperinvariant subspace problem can be reduced to that problem for perturbations of the bilateral shift. The product of commuting operators in $U_{p}$ is $U_{p}$, but this result is false in general. Quasisimilarity in $U_{e}$, is also studied; quasisimilar operators in $U_{e} \backslash U_{x}$ are unitarily equivalent modulo the ideal of compacts, and this result also holds in $U_{x_{0}}$ if the spectrum is also preserved.
\end{abstract}

1. Introduction. In this paper we study some subclasses of essentially normal operators. Let $L(H)$ be the algebra of all bounded linear operators on the complex separable Hilbert space $H$, and denote by $N(H), U(H)$ and $C(H)$ the set of normal, unitary and compact operators on $H$, respectively. Also let $\pi: L(H) \rightarrow L(H) / C(H)$ be the natural surjection onto the Calkin algebra. The set of essentially normal operators $N_{e}(H)$ is the set of $T \in L(H)$ such that $\pi(T)$ is normal, and the set of essentially unitary operators $U_{e}(H)$ is the set of $T \in L(H)$ such that $\pi(T)$ is unitary. In [1, Theorem 3.1, p. 71], the authors classify the operators in $U_{e}(H)$ as follows.

Proposition 1. If $T \in U_{e}(H)$, then $T=V+K$ where $K \in C(H)$ and $V$ is unitary, a shift of multiplicity $n$ or the adjoint of a shift of multiplicity $n$, according as $\operatorname{ind}(T)=0, \operatorname{ind}(T)=-n$ or $\operatorname{ind}(T)=n .($ Here, $\operatorname{ind}(T)$ denotes the Fredholm index on $T$; "shift" means unilateral shift.)

We shall also be interested in the following sets. For the integer $n$, let $U_{e}^{n}(H)=$ $\left\{T \in U_{e}(H): \operatorname{ind}(T)=n\right\}$. By Proposition $1, U_{e}^{0}(H)=U(H)+C(H)$ and $U_{e}(H)$ $=\bigcup\left\{U_{e}^{n}(H):-\infty<n<\infty\right\}$. Finally, for $1 \leqslant p<\infty$, let $C_{p}(H)$ be the Schatten $p$-class in $C(H)$ (see $\left[10\right.$, p. 102]). We put $C(H)=C_{\infty}(H)$. For $1 \leqslant p \leqslant \infty$ define $U_{p}(H)$ to be the set of all $T \in L(H)$ such that $I-T^{*} T \in C_{p}(H)$ and $\sigma(T)$ does not fill the open unit disc $D$.

Received by the editors December 17, 1981.

1980 Mathematics Subject Classification. Primary 47A15, 47A65, 47B30, 47C10.

Key words and phrases. Essentially unitary operator, compact operator, spectrum, essential spectrum, hyperinvariant subspace, shift, Fredholm operator, quasisimilar.

1984 American Mathematical Society $0002-9947 / 84 \$ 1.00+\$ .25$ per page 
Proposition 2. Let $1 \leqslant p \leqslant \infty$ and let $T \in L(H)$. Then $T \in U_{p}(H)$ iff $T \in$ $U(H)+C_{p}(H)$ and $\sigma(T)$ does not fill $D$.

Proof. Since the "if" part is clear, let $T \in U_{p}(H)$. Hence $\pi(T)$ is an isometry, and thus $\pi(T)$ is unitary since $\sigma(T)$ does not fill $D$. By Proposition $1, T=V+K$ with $K \in C(H)$ and $V$ unitary or a shift or the adjoint of a shift. Then $V$ is unitary since $\sigma(T)$ does not fill $D$. Hence ind $(T)=0$ and we may suppose that $T=W P$ with $W \in U(H)$ and $P^{2}=T^{*} T$. From the hypothesis $I-T^{*} T \in C_{p}(H)$ it follows that $I-P \in C_{p}(H)$. Hence $T=W P=W-W(I-P) \in U(H)+C_{p}(H)$.

COROLlary 1 . For $1 \leqslant p \leqslant \infty, U_{p}(H)$ is selfadjoint (closed under the map $T \rightarrow T^{*}$ ) and inverse closed. If $T \in U_{p}(H)$, then each $\lambda \in \sigma(T)$ with $|\lambda| \neq 1$ is an isolated eigenvalue of $T$.

Proof. The assertion on selfadjointness is immediate from Proposition 2. Let $T \in U_{p}(H)$ such that $T^{-1} \in L(H)$. Then $T^{-1}=V^{*}-T^{-1} K V^{*} \in U_{p}(H)$ where $K \in C_{p}(H)$ by Proposition 2. By [7, Proposition 1.27, p. 4], each $\lambda \in \sigma(T)$ with $|\lambda|>1$ is an isolated eigenvalue and $\sigma(T) \cap D$ is either $D$ or a countable set of isolated eigenvalues of $T$. Hence each $\lambda \in D \cap \sigma(T)$ is an isolated eigenvalue also.

The result cited in the last proof [7, Proposition 1.27] also yields the following

Corollary 2. If $T \in U_{e}^{0}(H) \backslash U_{\infty}(H)$, then $\sigma(\pi(T))$ (the essential spectrum) is the entire unit circle.

Proof. If $\lambda \notin \sigma(\pi(T))$ for some $|\lambda|=1$, then $D$ belongs to the connected complement of $\sigma(\pi(T))$; so $D \cap \sigma(T)$ is at most countable [7, Proposition 1.27]. Since $T=V+K$ with $V \in U(H)$ and $K \in C(H)$, by Proposition $2, T \in U_{\infty}(H)$. This contradicts the hypothesis $T \notin U_{\infty}(H)$, so the result follows.

Proposition 3. The following sets are uniformly closed in $L(H)$ : (i) $N(H)+C(H)$, (ii) $U_{e}(H)$ and (iii) $U_{e}^{n}(H)$ for each integer $n$.

Proof. Since (i) is proved in [1, Corollary 11.4, p. 119], we prove only (ii); the proof of (iii) is similar. Let $\left\{T_{m}\right\}$ be a sequence in $U_{e}(H)$ such that $T_{m} \rightarrow T$ uniformly. Since index is continuous, ind $\left(T_{m}\right)$ is some constant $r$ for sufficiently large $m$. By Proposition 1, $T \in U_{e}^{r}(H)$.

Proposition 4. For $1 \leqslant p \leqslant \infty, U_{p}(H)$ is uniformly dense in $U_{e}^{0}(H)$.

Proof. Clearly $U_{p}(H) \subset U_{q}(H)$ for $p<q$, so it suffices to prove that $U_{1}(H)$ is uniformly dense in $U_{e}^{0}(H)$. Moreover, since the latter set is closed by Proposition 3 , we need only show $U_{e}^{0}(H) \subset U_{1}(H)^{-}$. Let $T \in U_{e}^{0}(H)$ so that $T=V+K$ with $V \in U(H)$ and $K \in C(H)$. Let $\left\{K_{m}\right\}$ be a sequence of finite-rank operators converging uniformly to $K$. Clearly $\sigma\left(I+V^{*} K_{m}\right)$ is finite for each $m$, so we can choose a sequence of complex numbers $\left\{\alpha_{m}\right\}$ such that $\left|\alpha_{m}\right|=1$ and $\alpha_{m} \rightarrow 1$ and such that $T_{m}=V\left(I+\alpha_{m} V^{*} K_{m}\right)$ is invertible for each $m$. Clearly $T_{m} \in U_{1}(H)$ for each $m$ and $T_{m} \rightarrow T$. Hence $T \in U_{1}(H)^{-}$and the proof is complete. 
2. Transforms in $U_{e}(H)$. Recall that for $T \in L(H)$ and for a complex number $a$ such that $\|a T\|<1$ the Cayley transform $T_{a}$ is defined as $T_{a}=(T-a)(I-\bar{a} T)^{-1}$. A simple calculation (see [8, p. 240]) yields the identity

$$
I-T_{a}^{*} T_{a}=\left(1-|a|^{2}\right)\left(I-a T^{*}\right)^{-1}\left(I-T^{*} T\right)(I-\bar{a} T)^{-1}
$$

from which it follows that $T_{a}$ is unitary (resp., isometric, contractive) iff $T$ is unitary (resp., isometric, contractive). Moreover, we have

Proposition 5. If $T$ is a shift of multiplicity $n$, then $T_{a}$ is also such a shift for $|a|<1$.

Proof. By (*), $T_{a}$ is an isometry, hence by the Wold decomposition [8, p. 3], $H=M \oplus L$, where $M$ and $L$ are subspaces reducing $T_{a}$ such that $T_{a} \mid M$ is a shift and $T_{a} \mid L$ is unitary. Now $M$ and $L$ also reduce $T$, but since $T$ is irreducible either $M=(0)$ or $L=(0)$. Moreover, $T-a$ is bounded below and $\operatorname{dim}\left(\operatorname{ker}\left(T^{*}-\bar{a}\right)\right)=n$, hence ind $\left(T_{a}\right)=-n$ and thus $T_{a}=T_{a} \mid M$ is a shift of multiplicity $n$.

Theorem 1. Let $T \in U_{e}(H)$ and suppose that $T_{a}$ is defined for some $|a|<1$. Let $T=V+K$ be the decomposition given by Proposition 1 . Then $T_{a}=V_{a}+K_{a}$ where $V_{a}$ is the Cayley transform of $V$ and $K_{a} \in C(H)$ and

(i) $T_{a} \in U_{p}(H)$ iff $T \in U_{p}(H), 1 \leqslant p \leqslant \infty$;

(ii) $T_{a} \in U_{e}^{0}(H)$ iff $T \in U_{e}^{0}(H)$;

(iii) $V_{a}$ is a shift of multiplicity $n$ iff $V$ is.

Proof. Assume that the identity $T_{a}=V_{a}+K_{a}$ has been established. We note also that $T=\left(T_{a}\right)_{-\bar{a}}$. Hence (iii) results from Proposition 5 and (ii) from (*). Assertion (i) also follows from (*) and Proposition 2 and the fact that the map

$$
\lambda \rightarrow(\lambda-a)(1-\bar{a} \lambda)^{-1}
$$

is a homeomorphism of $D$.

To prove the representation $T_{a}=V_{a}+K_{a}$ we make the following computation:

$$
\begin{aligned}
T_{a} & =(V+K)_{a}=(V+K-a)(I-\bar{a} V-\bar{a} K)^{-1} \\
& =(V-a)(I-\bar{a} V-\bar{a} K)^{-1}+K_{1} \\
& =(V-a)(I-\bar{a} V)^{-1}(I-\bar{a} V)(I-\bar{a} V-\bar{a} K)^{-1}+K_{1} \\
& =V_{a} S+K_{1}
\end{aligned}
$$

where $K_{1} \in C(H)$ and

$$
\begin{aligned}
S & =(I-\bar{a} V)(I-\bar{a} V-\bar{a} K)^{-1} \\
& =(I-\bar{a} V)\left[(I-\bar{a} V)\left(I-\bar{a}(I-\bar{a} V)^{-1} K\right)\right]^{-1} \\
& =(I-\bar{a} V)\left(I-\bar{a}(I-\bar{a} V)^{-1} K\right)^{-1}(I-\bar{a} V)^{-1} \\
& =(I-\bar{a} V)\left(I+K_{2}\right)^{-1}(I-\bar{a} V)^{-1}
\end{aligned}
$$

with $K_{2} \in C(H)$. Since obviously $\left(I+K_{2}\right)^{-1}=I+K_{3}$ for some $K_{3} \in C(H), S=$ $I+K_{4}$ for $K_{4} \in C(H)$. So $T_{a}=V_{a}+K_{a}$ for $K_{a} \in C(H)$. 
Corollary 3. Let $T \in U_{e}(H)$ with $\operatorname{ind}(T) \neq 0$. If $T_{a}$ is defined, then $T_{a}$ is unitarily equivalent to a compact perturbation of $T$.

Proof. By (iii) of Theorem 1 and the fact that all shifts of the same multiplicity are unitarily equivalent $\left[8\right.$, p. 2], we have $T_{a}=V_{a}+K=W V W^{*}+K$ for $W \in$ $U(H)$. Hence $T_{a}=W T W^{*}+K^{\prime}$ for $K^{\prime} \in C(H)$.

REMARK. For $T \in U_{e}^{0}(H)$, Corollary 2 fails because the essential spectrum may not be preserved under the transform $T \rightarrow T_{a}$. (Of course, Corollary 2 also follows from Brown, Douglas and Fillmore [1, Theorem 11.1], if one shows that $\operatorname{ind}\left(T_{a}-\lambda\right)$ $=-n$ for $|\lambda|<1$.)

3. Path connectedness. In this section we prove that some of the sets introduced above are path connected. These results may be regarded as generalizations of the well-known facts that $U(H)$ and $N(H)$ are path connected. A path in $A \subset L(H)$ is a uniformly continuous map from $[0,1]$ into $A$. The set $A$ is path connected if every pair of elements in $A$ can be joined by a path in $A$. Every path connected set is connected.

THEOREM 2. The following sets are path connected:

(i) $N(H)+C(H)$;

(ii) $U_{p}(H)$ for $1 \leqslant p \leqslant \infty$;

(iii) the set of all contractions in $U_{p}(H)$;

(iv) $U_{e}^{n}(H)$ for each integer $n$.

Proof. Obviously for $T \in N(H)+C(H)$, the map $t \rightarrow P_{t}=t T, 0 \leqslant t \leqslant 1$, is a path in $N(H)+C(H)$ from the zero operator to $T$. Hence $N(H)+C(H)$ is path connected.

To prove (ii) let $1 \leqslant p \leqslant \infty$ and let $T \in U_{p}(H)$. It suffices to construct a path in $U_{p}(H)$ from $T$ to $I$. Suppose that $T$ is not invertible, so that the origin is isolated in $\sigma(T)$ by Corollary 2. There is some $r>0$ such that for $0 \leqslant t \leqslant r$ the map $t \rightarrow T_{t}$ (the Cayley transform) is a path of operators in $U_{p}(H)$ from $T=T_{0}$ to $T_{r}$ since $T_{t}$ is invertible for $t>0$. We may thus suppose that $T$ itself is invertible. In the polar decomposition $T=V P, V \in U(H)$ and $P$ is bounded below on $H$. Let $V_{t}(0 \leqslant t \leqslant 1)$ be a path from $V$ to $I$ in $U(H)$ [6, p. 102], and let $P_{t}=t I+(1-t) P(0 \leqslant t \leqslant 1)$. Clearly $P_{t}$ is invertible for $0 \leqslant t \leqslant 1$, and since $K=I-P \in C_{p}(H)$ (Proposition 2) and $P_{t}=I+(1-t) K$, it follows by Proposition 2 that $S_{t}=V_{t} P_{t}=V_{t}+(1-t) V_{t} K$ is a path in $U_{p}(H)$ from $T$ to $I$.

To see that the set of contractions in $U_{p}(H)$ is path connected, we note that since the Cayley transform of a contraction $T$ is also contractive (see $\$ 2$ ), by the last paragraph we may again suppose that $T$ is an invertible contraction. Then the positive part $P$ in the polar decomposition is also an invertible contraction. Hence the path $S_{t}=V_{t} P_{t}(0 \leqslant t \leqslant 1)$ constructed above also shows that (iii) holds.

For (iv) we distinguish three cases, $n=0, n<0$ and $n>0$. Let $T \in U_{e}^{0}(H)$ so that, by Proposition 1, $T=V+K$ with $V \in U(H)$ and $K \in C(H)$. Let $V_{t}(0 \leqslant t \leqslant 1)$ be any path from $V$ to $I$ in $U(H)$. Then $P_{t}=V_{t}+(1-t) K$ is a path in $U_{e}^{0}(H)$ from $T$ to $I$. Now let $n<0$ and let $T \in U_{e}^{n}(H)$. By Proposition $1, T=V+K$ where 
$K \in C(H)$ and $V$ is a shift of multiplicity $-n$. Since $P_{t}=V+(1-t) K$ is a path in $U_{e}^{n}(H)$ from $T$ to $V$, it is enough to show that every pair of shifts $V_{1}, V_{2}$ of multiplicity $-n$ can be joined by a path of such shifts. But $V_{1}$ and $V_{2}$ are unitarily equivalent [8, p. 2], so $V_{1}=W V_{2} W^{*}$ for some $W \in U(H)$. If $W_{t}(0 \leqslant t \leqslant 1)$ is a path in $U(H)$ from $W$ to $I$, then $Q_{t}=W_{t} V_{2} W_{t}^{*}$ is a path of shifts in $U_{e}^{n}(H)$ from $V_{1}$ to $V_{2}$. This proves that $U_{e}^{n}(H)$ is path connected for $n<0$. By taking adjoints the case for $n>0$ follows by the previous argument, so $U_{e}^{n}(H)$ is path connected for each $n$.

In [8, Chapter 8], the set of weak contractions is defined to be the set of contractions in $U_{1}(H)$. The following corollary is immediate from (iii) of Theorem 2.

COROllary 4. The set of weak contractions in $L(H)$ is path connected.

For $A \subset L(H)$ the similarity orbit $\operatorname{Sim}(A)$ of $A$ is the set of $T \in L(H)$ similar to some $S \in A$.

Proposition 6. If $A$ is path connected, then so is $\operatorname{Sim}(A)$.

Proof. Let $S, T \in A$ with $P_{t}(0 \leqslant t \leqslant 1)$ a path from $S$ to $T$ in $A$. Since $B S B^{-1}$ and $D T D^{-1}$ are in $\operatorname{Sim}(A)$ for invertible $B, D \in L(H)$ and since there is a path $Q_{t}$ $(0 \leqslant t \leqslant 1)$ of invertible operators from $B$ to $D, Q_{t} P_{t} Q_{t}^{-1}$ is a path in $\operatorname{Sim}(A)$ from $B S B^{-1}$ from $D T D^{-1}$.

COROllary 5. All sets (i)-(iv) in Theorem 2 have path connected similarity orbits.

4. Spectral continuity. We now consider the question of spectral continuity for some of the sets under study. The map $\sigma: L(H) \rightarrow$ Com into the set of compact sets in the plane given by $T \rightarrow \sigma(T)$ is a map between metric spaces if Com is given the Hausdorff metric. In general, this map is not continuous (see [3, p. 174]), but it may be continuous under certain restrictions, e.g. $\sigma$ is continuous on $N(H)$ [9]. The question of continuity may also be asked for other spectra, for example, the essential spectrum $\sigma_{e}(T)=\sigma(\pi(T))$ and the Weyl spectrum

$$
\sigma_{W}(T)=\bigcap\{\sigma(T+K) ; K \in C(H)\},
$$

as well as the spectral radius $r(T)=\sup \{|\lambda|: \lambda \in \sigma(T)\}$.

THEOREM 3. The essential spectrum $\sigma_{e}$ is continuous on $N_{e}(H)$, and the Weyl spectrum is continuous on $N(H)+C(H)$.

Proof. Let $\left\{T_{m}\right\}$ be a sequence in $N_{e}(H)$ converging uniformly to $T \in N_{e}(H)$. Then $\pi\left(T_{m}\right) \rightarrow \pi(T)$ in the set of normal operators in the Calkin algebra. By the remark above $\sigma_{e}\left(T_{m}\right) \rightarrow \sigma_{e}(T)$, so $\sigma_{e}$ is continuous on $N_{e}(H)$. For $T \in N(H)+C(H)$ it is known that $\sigma_{e}(T)=\sigma_{W}(T)$, hence the second statement of the theorem follows from the first by restriction of $\sigma_{e}$ to $N(H)+C(H)$.

THEOREM 4. The spectrum $\sigma$ is continuous on $U_{\infty}(H)$.

Proof. Let $T_{m} \rightarrow T$ uniformly in $U_{\infty}(H)$. By Theorem 3, $\sigma_{W}\left(T_{m}\right) \rightarrow \sigma_{W}(T)$, hence by Corollary 1 and the proof of [3, Proposition 3.7], $\sigma\left(T_{m}\right) \rightarrow \sigma(T)$. 
EXAMPLE 1. We show that $\sigma$ is not continuous on $U_{e}^{0}(H)$. Let $V$ be the bilateral shift of multiplicity 1 and let $K$ be the rank-one operator such that $T_{1}=V+K=S$ $\oplus S^{*}$ where $S$ is the (unilateral) shift of multiplicity $1\left[6\right.$, p. 295]. Hence $\sigma\left(T_{1}\right)=D^{-}$ but if $T_{\lambda}=V+\lambda K, 0 \leqslant \lambda<1$, it is easy to see that $\sigma\left(T_{\lambda}\right)$ is the unit circle. Hence $\sigma\left(T_{\lambda}\right)$ does not converge to $\sigma\left(T_{1}\right)$, while obviously $T_{\lambda} \rightarrow T_{1}$. This shows that $\sigma$ is not continuous on $U_{e}^{0}(H)$.

THEOREM 5. The spectral radius is continuous on $U_{e}(H)$.

Proof. Let $T_{k} \rightarrow T$ uniformly in $U_{e}(H)$. By the proof of Proposition 3 we may suppose that all $T_{k}$ (and hence $T$ ) lie in some $U_{e}^{n}(H)$ for a fixed integer $n$. Let $\varepsilon>0$. Since $r$ is upper semicontinuous [6, p. 86], $r\left(T_{k}\right) \leqslant r(T)+\varepsilon$ for $k$ sufficiently large. If $r(T)=1$, then $r(T) \leqslant r\left(T_{k}\right)$ for all $k$, hence $r\left(T_{k}\right) \rightarrow r(T)$. If $r(T)>1$, then $r(T)=|\lambda|$ for some isolated $\lambda \in \sigma(T)$. By [3, Lemma 1.5], the $\varepsilon$-neighborhood of $\lambda$ contains a point of $\sigma\left(T_{k}\right)$ for $k$ large, so for each such $k, r\left(T_{k}\right) \geqslant r(T)-\varepsilon$. Again $r\left(T_{k}\right) \rightarrow r(T)$.

REMARK. In fact, Theorem 5 is a corollary of the following. Every $T \in U_{e}(H)$ is a point of continuity of $r$. This statement follows in turn from Proposition 1 and Conway and Morrell [3, Theorem 2.6].

5. Invariant subspaces. It is not known whether every $T \in N_{e}(H)$ has a nontrivial invariant subspace; in particular, the question is open for $T$ in $N(H)+C(H)$. (For $T \in N_{e}(H) \backslash[N(H)+C(H)]$ the answer is yes; see [1, p. 118].) In [10, Chapter 6], Radjavi and Rosenthal prove that if $1 \leqslant p<\infty$, then $T \in U_{p}(H)$ has a nontrivial invariant subspace. Moreover, if $T \in U_{e}^{(0}(H)$ has $\lambda \in \sigma(T)$ with $|\lambda| \neq 1$, then $\lambda$ is an eigenvalue of $T$ by Weyl's theorem. Hence the question whether every $T \in U_{e}(H)$ has a nontrivial invariant subspace reduces to this: if $T \in U_{\infty}(H)$ and $\sigma(T)$ is contained in the unit circle, does $T$ have a nontrivial invariant subspace? Below we give conditions sufficient for such $T$ to have a nontrivial hyperinvariant subspace, i.e. one invariant under the commutant of $T$. In several of these results we apply Lomonosov's lemma [10, Theorem 8.24, p. 158], which states that every $T \in L(H)$ commuting with a nonzero $K \in C(H)$ has a nontrivial hyperinvariant subspace.

Theorem 6. Let $T \in U_{\infty}(T)$ with $\sigma(T)$ contained in the unit circle. Let $R_{T}$ be the uniform closure in $L(H)$ of all rational functions in $T$. If $R_{T}+C(H)$ is uniformly closed in $L(H)$, then either $T$ has a nontrivial hyperinvariant subspace or $T$ is a scalar.

Proof. If $R_{T} \cap C(H) \neq(0)$, then $T$ has a nontrivial hyperinvariant subspace by Lomonosov's lemma. If $R_{T} \cap C(H)=(0)$, then it is easy to see that $\pi$ is bounded below on $R_{T}$, hence there exists $M>0$ such that for every rational function $f$ with poles off $\sigma(T)$

$$
\|f(T)\| \leqslant M\|\pi(f(T))\| \leqslant M\|f\|_{\infty}
$$

where $\|f\|_{\infty}=\sup \{|f(\lambda)|:|\lambda|=1\}$ and the second inequality holds because $\pi(f(T))$ is normal. In particular, for $|\lambda| \neq 1$

$$
\left\|(\lambda-T)^{-1}\right\| \leqslant M /|1-| \lambda|| .
$$


By the theory of $A$-unitary operators [2, Chapter 5], $T$ is "decomposable" and hence has a nontrivial hyperinvariant subspace if $\sigma(T)$ has more than one point $[\mathbf{2}$, Chapter 2]. If $\sigma(T)=\{\lambda\}$, then $\pi(\lambda-T)$ is a normal quasinilpotent operator in the Calkin algebra, so $\pi(\lambda-T)=0$. Hence $\lambda-T \in C(H)$ and $\lambda-T=0$. This proves the theorem.

Theorem 7. Let $T \in N_{e}(H)$ such that $R_{T}$ (see Theorem 6) contains a nonzero quasinilpotent operator. Then $T$ has a nontrivial hyperinvariant subspace.

Proof. Let $Q$ be a nonzero quasinilpotent operator in $R_{T}$. By Lomonosov's lemma it is enough to prove $Q \in C(H)$. There is a sequence $\left\{f_{n}(T)\right\}$ of rational functions in $T$ converging uniformly to $Q$, so $\pi(Q)$ is the limit of normal operators in the Calkin algebra. Then $\pi(Q)$ is normal and quasinilpotent, and thus $\pi(Q)=0$, i.e. $Q \in C(H)$.

COROllary 6. Let $T \in U_{\infty}(H)$ with $\sigma(T)$ contained in the unit circle and such that the range of $R_{T}$ under the Gelfand representation $g$ is closed. Then $T$ has a nontrivial hyperinvariant subspace.

Proof. If ker $g \neq(0)$, then $R_{T}$ contains a nonzero quasinilpotent operator and the result follows by Theorem 7 . If ker $g=(0)$, then it follows that $g$ is an isomorphism, hence $(* *)$ in the proof of Theorem 6 holds for some $M>0$ and all $|\lambda| \neq 1$. The proof ends like that of Theorem 6 .

The next result relates the ideas of this and the preceding section.

THEOREM 8. Let $T \in N_{e}(H)$ and let $T$ be a point of continuity of the spectrum. Then either $T$ has a nontrivial hyperinvariant subspace or $T$ is a scalar.

Proof. If ind $(\lambda-T) \neq 0$ for some $\lambda$, then either $T$ or $T^{*}$ has an eigenvalue of finite multiplicity so in this case $T$ has a nontrivial hyperinvariant subspace. If $\operatorname{ind}(\lambda-T)=0$ for all $\lambda \notin \sigma_{e}(T)$, then $T \in N(H)+C(H)$ by [1, Corollary 11.2]. We may also suppose $\sigma(T)$ to be connected, otherwise apply the Riesz functional calculus to produce a nontrivial hyperinvariant subspace. By [3, Corollary 3.2], the set $\{\lambda \in \sigma(T): \operatorname{ind}(\lambda-T)=0\}$ is empty, so $\sigma_{e}(T)=\sigma(T)$. Let $\lambda \in \sigma(T)$ so that by [3, Theorem 3.1 and Proposition 1.3] every neighborhood of $\lambda$ contains a (connected) component of $\sigma_{e}(T)$. It follows that $\sigma(T)=\{\lambda\}$, so $\lambda-T$ is quasinilpotent. If $\lambda-T \neq 0$, then $T$ has a nontrivial hyperinvariant subspace by Theorem 7, otherwise $T=\lambda$.

We now show that the hyperinvariant subspace problem for operators in $U_{e}(H)$ can be reduced to that same question for perturbations of the bilateral shift.

THEOREM 9. Let $T \in U_{e}(H)$ and let $B$ be a bilateral shift of multiplicity 1 on some orthonormal basis in $H$. If for each $K \in C(H)$ the perturbation $B+K$ has a nontrivial hyperinvariant subspace, then $T$ does also or $T$ is a scalar.

Proof. By the remarks at the beginning of this section and by those of Pearcy in [7, p. 47], we may suppose that $\sigma(T)$ is connected and $\sigma(T)=\sigma_{e}(T)$. If $\sigma(T)=\{\lambda\}$, then either $T=\lambda$ or $T$ has a nontrivial hyperinvariant subspace by Theorem 7 . If 
$\sigma(T)$ has more than one point, then it is an arc on the unit circle. By the spectral mapping theorem there is an integer $j$ such that $\sigma_{e}\left(T^{j}\right)$ is the entire circle. Thus $\sigma_{e}(B)=\sigma_{e}\left(T^{j}\right)$, and since $\lambda-T^{j}$ and $\lambda-B$ are both invertible for $\lambda \notin \sigma_{e}(B)$ it follows from [1, Theorem 11.1], that $V T^{j} V^{*}=B+K$ for some $V \in U(H)$ and $K \in C(H)$. By hypothesis $B+K$ has a nontrivial hyperinvariant subspace and thus $T^{j}$ also has such a subspace, say $M$. Clearly $M$ is $T$-invariant, and if $S$ commutes with $T$ it also commutes with $T^{j}$. Hence $M$ is $S$-invariant and is thus hyperinvariant for $T$.

We now recall that $T \in L(H)$ has the single-valued extension property (svep) [4, p. 3], if the zero function is the only analytic map $f: G \rightarrow H$ ( $G$ open in the plane) such that $(\lambda-T) f(\lambda)=0$ for $\lambda \in G$. One sees easily that $T$ has the svep if $\sigma(T)$ is nowhere dense, hence every $T \in U_{p}(H), 1 \leqslant p<\infty$, has the svep. The svep is also preserved under restriction to an invariant subspace. Let $M$ be invariant for $T$. Denote by $T \mid M$ the restriction of $T$ to $M$ and by $T^{M}$ the operator induced by $T$ on $H / M$, and denote by $M^{\perp}$ the annihilator of $M$ in $H$.

Theorem 10. Let $T \in U_{p}(H), 1 \leqslant p \leqslant \infty$, and let $M$ be invariant. Then the following assertions are equivalent:

(1) $T \mid M \in U_{p}(M)$.

(2) $T^{M} \in U_{p}(H / M)$.

(3) $T^{*} \mid M \in U_{p}\left(M^{\perp}\right)$.

(4) $\sigma(T \mid M) \subset \sigma(T)$.

(5) $\sigma\left(T^{M}\right) \subset \sigma(T)$.

(6) $M$ is invariant under all $(\lambda-T)^{-1}, \lambda \notin \sigma(T)$.

(7) $M$ is invariant under some $(\lambda-T)^{-1}, \lambda \in D \backslash \sigma(T)$.

(8) $(\lambda-T) M=M$ for some $\lambda \in D$.

(9) $\operatorname{ind}(T \mid M)=0$.

Proof. We note first that (4), (5) and (6) are equivalent by [4, Proposition 1.15]; and by Corollary 1 and the identification of $(T \mid M)^{*}$ with $T^{*} \mid M^{\perp},(2)$ and (3) are equivalent. Let $T_{1}=T \mid M$ and $T_{2}=T^{M}$. By looking at matrix representations we see that $I-T_{1}^{*} T_{1} \in C_{p}(M)$ and $I-T_{2}^{*} T_{2} \in C_{p}(H / M)$, hence (4) $\Rightarrow(1)$ and (5) $\Rightarrow$ (2).

We next prove that $(1) \Rightarrow(7)$. If (1) holds and (7) fails, then $\sigma(T)$ separates the plane. Moreover, by [4, Proposition 1.17], $D \subset \sigma(T)$, which contradicts the hypothesis, so $(1) \Rightarrow(7)$. Also $(7) \Rightarrow(6)$ by [4, Proposition 1.17]. Now use the equivalence (1) $\Leftrightarrow(5)$ to deduce (3) $\Rightarrow(5)$. We thus have (1)-(7) equivalent.

Clearly $(4) \Rightarrow(8)$. We noted above that $T_{1}$ have the svep since $T$ does, so $(8) \Rightarrow(7)$ by $[5$, p. 62].

By Proposition 2, (1) $\Rightarrow(9)$. Conversely, if (9) holds then since $\pi\left(T_{1}\right)$ is unitary it follows by Proposition 1 that $T_{1}=V+K$ with $V \in U(M)$ and clearly $K \in C_{p}(M)$. If $\sigma\left(T_{1}\right)$ fills $D$, then by Weyl's theorem each $\lambda \in D$ is an eigenvalue of $T_{1}$. By Finch [5, p. 65], $T_{1}$ does not have the svep, and this contradiction proves that $\sigma\left(T_{1}\right)$ does not fill $D$. By Proposition 2, $T_{1} \in U_{p}(M)$, and the proof is complete. 
Corollary 7. Let $T \in U_{p}(H), 1 \leqslant p \leqslant \infty$, and let $M$ be invariant. Then $T \mid M \in$ $U_{p}(M)$ if one of the following holds: (i) $M$ reduces $T$, (ii) $M$ is hyperinvariant, (iii) $\sigma(T)$ does not separate the plane, (iv) $\left(\lambda_{0}-T\right) M$ is dense in $M$ for some $\lambda_{0} \in D$.

Proof. In cases (i)-(iii), $M$ is invariant under $(\lambda-T)^{-1}$ for all $\lambda \notin \sigma(T)$. In case (iv) we observe that $\lambda-T$ is Fredholm for each $\lambda \in D$, hence $\lambda-T$ has closed range. It follows that $(\lambda-T) M$ is closed for all $\lambda \in D$. If $\left(\lambda_{0}-T\right) M$ is dense in $M$, then $\lambda_{0}-T$ is surjective on $M$. By (8) of Theorem $9, T \mid M \in U_{p}(M)$.

6. Other properties. First we compare the class $N(H)+C(H)$ to the class of normal operators. It is well known that every normal operator with a right or left inverse is invertible. This result extends to $N(H)+C(H)$.

THEOREM 11. If $T \in N(H)+C(H)$ has a left or right inverse, then $T$ is invertible.

Proof. If $S T=I$ for some $S \in L(H)$, then $\operatorname{ker} T=(0)$; and since $\pi(S) \pi(T)=I$ and $\pi(T)$ is normal, $\pi(T)$ has an inverse in the Calkin algebra. But clearly $\operatorname{ind}(T)=0$, so $T$ is surjective on $H$. By the closed graph theorem $T$ is invertible. If $T S=I$ for some $S$, since $T^{*} \in N(H)+C(H)$ also, we can take adjoints and apply the above reasoning to $S^{*} T^{*}=I$.

Corollary 8. For $T \in N(H)+C(H)$ the approximate point spectrum $\sigma_{a}(T)$ coincides with $\sigma(T)$.

Proof. Suppose that $\lambda \in \sigma(T) \backslash \sigma_{a}(T)$. Since $T=N+K$ with $N \in N(H)$ and $K \in C(H)$ and $\lambda-T$ is bounded below on $H, \lambda-T \in N(H)+C(H)$ and $\lambda-T$ has a left inverse in $L(H)$. By Theorem 9 we get the contradiction $\lambda \notin \sigma(T)$, hence $\sigma(T)=\sigma_{a}(T)$.

The standard shift $V$ shows that neither Theorem 9 nor Corollary 8 is valid for $N_{e}(H)$ because $V^{*} V=I$ but $V$ is not invertible and $\sigma(V)=D^{-}$while $\sigma_{a}(T)$ is the unit circle.

Obviously $U(H)$ is closed under (operator) multiplication, and it is easy to see that $U_{e}^{0}(H)=U(H)+C(H)$ and $U_{e}(H)$ are also multiplicatively closed. On the other hand, $U_{p}(H)$ is not multiplicatively closed for any $1 \leqslant p \leqslant \infty$. Let $T_{1}=V+K$ in Example 1 above. Both $V$ and $I+V^{*} K$ are in $U_{1}(H)$, but their product $T_{1} \notin U_{\infty}(H)$. As in the case of normal operators, the result is affirmative for commuting operators.

TheOREM 12. For $1 \leqslant p, r \leqslant \infty$ let $T \in U_{p}(H)$ and $S \in U_{r}(H)$. If $T S=S T$, then $T S \in U_{m}(H)$ where $m=\max (p, r)$.

Proof. Clearly $T S \in U(H)+C_{m}(H)$, so by Proposition 2 it suffices to prove that $\sigma(T S)$ does not fill $D$. Since $D \cap[\sigma(T) \cup \sigma(S)]$ is at most countable, we can find a sequence of nonzero complex numbers $\left\{\alpha_{n}\right\} \subset D \backslash[\sigma(T) \cup \sigma(S)]$ such that $\alpha_{n} \rightarrow 0$. For all $n$ the Cayley transforms $T_{n}=T_{\alpha_{n}}$ and $S_{n}=S_{\alpha_{n}}$ are invertible, hence $T_{n} S_{n} \in U_{m}(H)$. By Corollary $1, \sigma\left(T_{n} S_{n}\right)$ is nowhere dense, so $T_{n} S_{n}$ has the svep for all $n$. But $T_{n} S_{n}$ commutes with $T S$, hence $T S$ also has the svep by [4, p. 9]. If $\sigma(T S)$ 
fills $D$, then since $T S$ is Fredholm it does not have the svep by [5, p. 65]. This contradiction proves the theorem.

Proposition 7. Let $T, S \in N_{e}(H)$ such that $\pi(T S)=\pi(S T)$. Then $T S$ and $T+S$ are both in $N_{e}(H)$.

Proof. Since $\pi(T)$ and $\pi(S)$ are commuting normal operators in the Calkin algebra, the result follows frum Fuglede's theorem.

Corollary 9. Let $T, S \in N(H)+C(H)$ such that $\pi(T S)=\pi(S T)$. If $\sigma(T S)$ is nowhere dense, then $T S \in N(H)+C(H)$.

Proof. By Proposition 7, TS $\in N_{e}(H)$. Since $\sigma(T S)$ is nowhere dense, the set $\{\lambda$ : $\operatorname{ind}(\lambda-T S) \neq 0\}$ is empty, hence $T S \in N(H)+C(H)$ by [1, Corollary 11.2].

We now discuss $U_{e}(H)$ relative to quasisimilarity. Recall that $T, S \in L(H)$ are quasisimilar if there exist $A, B \in L(H)$ with trivial kernels and dense ranges such that $T A=A S$ and $B T=S B$. If $T, S$ are quasisimilar unitary operators, then $T$ and $S$ are unitarily equivalent [8, p. 71]. Also, put $n(\lambda-T)=\operatorname{dim}(\operatorname{ker}(\lambda-T))$.

Proposition 8. If $T, S \in L(H)$ are quasisimilar, then $n(\lambda-T)=n(\lambda-S)$ for all $\lambda$.

Proof. Let $A$ be as above. Then $A(\lambda-S)=(\lambda-T) A$ for all $\lambda$ and

$$
(\lambda-T) A(\operatorname{ker}(\lambda-S))=A(\lambda-S) \operatorname{ker}(\lambda-S)=(0),
$$

hence $A(\operatorname{ker}(\lambda-S)) \subset \operatorname{ker}(\lambda-T)$. Since $A$ is injective $n(\lambda-S) \leqslant n(\lambda-T)$, and the result follows by symmetry.

Corollary 10. Let $T, S \in N_{e}(H)$ be quasisimilar. If $\sigma_{e}(T)=\sigma_{e}(S)$, then $T=$ $W S W^{*}+K$ with $W \in U(H)$ and $K \in C(H)$.

Proof. By [1, Theorem 11.1], we need only show that ind $(\lambda-T)=\operatorname{ind}(\lambda-S)$ for $\lambda \notin \sigma_{e}(T)$, but this follows from Proposition 8 .

TheOREM 13. Let $T, S \in U_{e}(H)$ be quasisimilar. Then $T=W S W^{*}+K$ with $W \in U(H)$ and $K \in C(H)$ if any one of the following holds:

(i) both $T$ and $S \notin U_{\infty}(H)$;

(ii) $T, S \in U_{\infty}(H)$ and $\sigma(S)=\sigma(T)$;

(iii) $T$ and $S$ are both power bounded and $\sigma(T)$ and $\sigma(S)$ are contained in the unit circle (i.e. $\left\|T^{n}\right\| \leqslant M$ for some $M>0$ and all integers $n$ ).

Proof. By Corollary 10 we need to prove that $\sigma_{e}(T)=\sigma_{e}(S)$ follows from each of (i)-(iii).

If (i) holds, then $\sigma_{e}(S)$ and $\sigma_{e}(T)$ are both the unit circle by Corollary 2. In case (ii) we must show that any isolated $\lambda \in \sigma(T)$ such that $|\lambda|=1$ is simultaneously in $\sigma_{e}(T)$ and $\sigma_{e}(S)$. For such a $\lambda$, let $M$ and $N$ be the respective ranges of the Riesz projections of $T$ and $S$ associated with the "spectral" set $\{\lambda\}$. By Corollary 7 , $T \mid M \in U_{\infty}(M)$ and $S \mid N \in U_{\infty}(N)$. Hence $T \mid M=\lambda_{M}+K_{1}$ and $S \mid N=\lambda_{N}+K_{2}$, where $\lambda_{M}$ is the scalar $\lambda$ on $M$ and $K_{1}, \mathrm{~K}_{2}$ are compact. It thus suffices to prove that $\operatorname{dim} M=\operatorname{dim} N=\infty$. Let $A \in L(H)$ be an operator effecting the quasisimilarity 
such that $A T=S A$. Then $(A M)^{-}$is $S$-invariant and it follows from the quasisimilarity of $T$ and $S$ that $\sigma\left(S \mid(A M)^{-}\right)=\{\lambda\}$ and, since $N$ is a spectral maximal space for $S$ [2, p. 19], we get $A M \subset N$. But $A$ is injective, hence $\operatorname{dim} M \leqslant \operatorname{dim} N$. By symmetry we obtain $\operatorname{dim} M=\operatorname{dim} N$, and the result follows.

Finally, suppose that (iii) holds. Since $T$ and $S$ are power bounded, it is well known that $T$ and $S$ are, respectively, similar to unitary operators $V$ and $W$. But similar operators are quasisimilar and quasisimilarity is an equivalence relation, hence $V$ and $W$ are quasisimilar. By the remark above, $V=X W X^{*}$ for $X \in U(H)$. Moreover, similarity clearly preserves essential spectrum, so $\sigma_{e}(T)=\sigma_{e}(V)=\sigma_{e}(W)$ $=\sigma_{e}(S)$, and the proof is complete.

\section{REFERENCES}

1. L. G. Brown, R. G. Douglas and P. A. Fillmore, Unitary equivalence modulo the compact operators and extensions of $C^{*}$-algebras (Proc. Conf. Operator Theory), Lecture Notes in Math., vol. 345, SpringerVerlag, Berlin and New York, 1973.

2. I. Colojoara and C. Foias, Theory of generalized spectral operators, Gordon \& Breach, New York, 1968.

3. J. Conway and B. Morrell, Operators that are points of spectral continuity, Integral Equations Operator Theory 2 (1979), 174-198.

4. I. Erdelyi and R. Lange, Spectral decompositions on Banach space, Lecture Notes in Math., vol. 623, Springer-Verlag, Berlin and New York, 1977.

5. J. Finch, The single-valued extension property on a Banach space, Pacific J. Math. 58 (1975), 61-69.

6. P. R. Halmos, A Hilbert space problem book, Van Nostrand, Princeton, N. J., 1967.

7. C. Pearcy, Some recent developments in operator theory, CBMS Regional Conf. Ser. in Math., no. 36, Amer. Math. Soc., Providence, R. I., 1978.

8. B. Sz.-Nagy and C. Foias, Harmonic analysis of operators on Hilbert space, North-Holland, Amsterdam, 1970.

9. J. D. Newburgh, The variation of spectra, Duke Math. J. 18 (1951), 165-176.

10. H. Radjavi and P. Rosenthal, Invariant subspaces, Springer-Verlag, Berlin and New York, 1973.

Department of Mathematics, University of New Orleans, New Orleans, Louisiana 70148

Current address: Department of Mathematics, Central Michigan University, Mt. Pleasant, Michigan 48859 